\title{
Protecting Encapsulin Nanoparticles with Cysteine-Knot Miniproteins
}

\author{
Robin Klem, ${ }^{\dagger}$ Mark V. de Ruiter, ${ }^{\dagger}$ and Jeroen J.L.M. Cornelissen*(i) \\ Laboratory for Biomolecular Nanotechnology, MESA+ Institute for Nanotechnology, University of Twente, 7500 AE Enschede, The \\ Netherlands
}

\section{Supporting Information}

ABSTRACT: A big hurdle for the use of protein-based drugs is that they are easily degraded by proteases in the human body. In an attempt to solve this problem, we show the possibility to functionalize TM encapsulin nanoparticles with an mEETI-II knottin miniprotein from the cysteine-stabilized knot class. The resulting particles did not show aggregation and retained part of their protease inhibitive function. This imposes a protection toward protease, in this case, trypsin, degradation of the protein cage. The used chemistry is easy to apply and thus suitable to protect other protein systems from degradation. In addition, this proof of

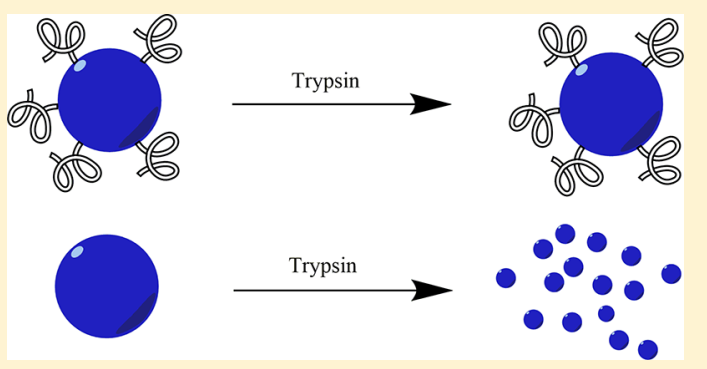
principle opens up the use of other knottins or cysteine-stabilized knots, which can be attached to protein cages to create a heterofunctionalized protein nanocage. This allows specific targeting and tumor suppression among other types of functionalization. Overall, this is a promising strategy to protect a protein of interest which brings oral administration of protein-based drugs one step closer.

KEYWORDS: bacterial nanocompartment, protease inhibitor, nanocage, antibody substitute

\section{INTRODUCTION}

Protein-based formulations are more and more used in drug applications because of their high efficiency and biocompatibility. They are applied as recombinant protein vaccines ${ }^{1}$ and treatment for cancer ${ }^{2}$ and show promise for the treatment of various other diseases. ${ }^{3}$ One of the major drawbacks of proteins as drugs is the reduced bioavailability, in part because these get degraded by proteases. To protect the proteins from degradation, they can be functionalized with polymers such as polyethylene glycol (PEG), which can increase efficacy, but they still suffer various drawbacks related to the specificity of glycosylation. ${ }^{4}$ To address the issue of protease-based protein degradation more specifically, we seek to improve the stability of proteins using a new strategy based on knottins.

Knottins are a class of miniproteins that are natural enzymeinhibitor toxins. Their defined cysteine-stabilized knot structures impose a high thermal stability as well as protection from proteases ${ }^{5}$ and allow a great degree of freedom to modify their loops. ${ }^{6}$ The modified loop peptide sequence can be used for high affinity binding onto specific receptors and proteins, which exceed the binding affinities of free peptides. Therefore, these short peptides hold great promise as stable and cheap antibody substitutes. A multitude of knottins and their derivatives have been developed or found in nature and are combined in a database. ${ }^{7,8}$ They function as analgesics, ${ }^{9,10}$ antimalarials, ${ }^{11}$ and pesticides. ${ }^{12}$ Knottins are not immunogenic $^{6}$ and can function in a variety of medical ways: as anticancer drugs, antiviral drugs, and in vivo imaging agents. $^{13,14}$
Knottins can also be used to inhibit specific proteins, such as trypsin, which is inhibited by the Ecballium elaterium trypsin inhibitor II (EETI-II) knottin, which can be modified while maintaining its structure. ${ }^{15}$ The EETI-II knottins have three specific loops, and one of the loops has a specific amino acid sequence that probably blocks the active site of trypsin using competitive inhibition. ${ }^{16}$ However, Sankaran et al. show, using a kinetic analysis, that the inhibition is a mix of competitive and noncompetitive inhibition. ${ }^{13}$ This inhibition has an $\sim 10$ $\mathrm{nM}$ affinity, ${ }^{17}$ which is caused by the specific amino acid sequence combined with the specific shape of the loop that is formed by the cysteines in the knottin. ${ }^{18}$ This makes EETI-II a suitable candidate in our study, given that trypsin is a main protease component of the digestive system of many vertebrates. Thus, trypsin is an important target in order to protect the protein of interest.

Protein-based nanocages are a class of protein materials that can benefit from such extra protection. These protein nanoparticles can function as biocompatible and biodegradable cellular-delivery platforms that can be used for the delivery of, for example, active small molecules, genes, ${ }^{19}$ proteins, ${ }^{20}$ and nanoparticles. ${ }^{21}$ Protein cages include viruses, ferritins, and recently discovered bacterial nano and microcompartments,

Special Issue: Click Chemistry for Medicine and Biology

Received: June 15, 2018

Revised: July 9, 2018

Accepted: July 12, 2018

Published: July 26, 2018 
such as encapsulins. Encapsulins derived from Thermotoga maritima (TM) are an especially interesting protein cage, because these remain stable at high temperatures and over a wide $\mathrm{pH}$ range. TM encapsulins are icosahedral particles of 22 nm with a $T=1$ symmetry, consisting of 60 capsid protein (CP) monomers, ${ }^{22}$ which can load foreign cargo. ${ }^{23}$ On each of these monomers, one cysteine group is solvent exposed, allowing specific modifications that can alter the encapsulin exterior and functionalize it in a controlled and defined manner. ${ }^{24}$

In this study, we genetically modify the EETI-II knottin to create a flexible and accessible $\mathrm{N}$-terminus, and we replace the only lysine in the native knottin with a serine to avoid binding to other binding sites. We connected this knottin to TM encapsulins with a heterofunctional linker (EMCS) to create a functionalized protein nanocage with trypsin inhibitive properties.

\section{EXPERIMENTAL SECTION}

Materials. Chemicals were purchased from Sigma-Aldrich unless stated otherwise. Milli-Q water was obtained by ultrafiltration (Millipore Adv. A10, $18 \mathrm{M} \Omega \cdot \mathrm{cm}$ at $25{ }^{\circ} \mathrm{C}$ ).

Equipment. UV-vis measurements to determine protein and DNA concentrations were performed using a Thermo Scientific Nanodrop 1000 and with a PerkinElmer Lambda 850 spectrometer. Standard quartz cuvettes with a $1 \mathrm{~cm}$ path length were used. Kinetic absorption measurements for trypsin inhibitor assays were performed using a TECAN Infinite 200 Pro plate reader. For transmission electron microscopy (TEM), samples ( $5 \mu \mathrm{L})$ were applied onto Formvar-carbon coated grids. After $1 \mathrm{~min}$, the excess of liquid was drained using filter paper. Uranyl acetate $(5 \mu \mathrm{L}, 1 \% \mathrm{w} / \mathrm{v})$ was added, the excess of liquid was drained after $15 \mathrm{~s}$, and the samples were dried for $30 \mathrm{~min}$ at room temperature. Imaging was performed on an FEG-TEM (Phillips CM 30) operated at $300 \mathrm{kV}$ acceleration voltages. Dynamic light scattering (DLS) measurements were performed using a Microtrac Nanotrac Wave W3043. The viscosity and refractive index of water and the refractive index of proteins (1.54) were used. Matrix assisted laser desorption/ionization time-of-flight (MALDI-TOF) analysis was performed with a Waters MALDI SYNAPT G1 high definition mass spectrometer on a sinapic acid matrix.

Purification of Thermotoga maritima Encapsulin. TM encapsulins were produced and purified using the method described by Rurup et al. ${ }^{25}$ To purify TM encapsulins instead of Brevibacterium linens encapsulins, after RNase addition, we ultracentrifuged the sample at $234000 \mathrm{~g}$ for $17 \mathrm{~h}$ at $10{ }^{\circ} \mathrm{C}$ and subsequently dissolved the pellet in $1 \mathrm{~mL}$ of encapsulin buffer (20 mM Trizma base, $150 \mathrm{mM} \mathrm{NH} \mathrm{m}_{4} \mathrm{Cl}, 20 \mathrm{mM} \mathrm{MgCl}, 1 \mathrm{mM}$ $\beta$-mercaptoethanol, $\mathrm{pH} 7.5)$.

Fluorophore Binding on TM. TM encapsulins were rebuffered to $0.1 \times$ PBS buffer with $0.1 \%(\mathrm{v} / \mathrm{v})$ TWEEN-20 using Zeba Spin 7k MWCO Desalting Columns (Thermo Fisher). Oregon Green 488 maleimide dye was dissolved in DMSO at $1 \mathrm{mg} / \mathrm{mL}$ and mixed with $\mathrm{TM}$ encapsulins in a 1:120 ratio (encapsulin/Oregon Green 488). The reaction was incubated for $1 \mathrm{~h}$ at $21{ }^{\circ} \mathrm{C}$ and purified twice using the Zeba spin column.

Molecular Cloning of (m)EETI-II Constructs. The molecular cloning of (m)EETI-II constructs was performed similar to a protocol described by Sankaran et al. ${ }^{1 \frac{1}{3}}$ but with several modifications. ssDNA sequences corresponding to the $\beta$-trypsin inhibitor knottins were ordered from Eurofins MWG
Operon, Germany. The 3'-terminal contained a stop codon. All genetic sequences, plasmids, and peptide sequences are provided in Figure S1. The genetic constructs had $5^{\prime}$ BsrGI and $3^{\prime}$ NheI restriction sites, which were used to insert the gene behind the gene for TFP through restriction digestion enzymes on the suppliers' and the pET-15b plasmid. The samples were purified with agarose gel electrophoresis, using Wizard SV Gel and a PCR Clean-Up System (Promega) followed by ligation with T4 DNA ligase (New England Biolabs Inc.). The resulting pET15b TFP-knottin plasmids were transformed to NovaBlue ultracompetent cells (Novagen) and grown overnight on LB agar plates containing 100 $\mathrm{mg} / \mathrm{L}$ ampicillin. Plasmids were extracted from individual colonies using a Qiagen spin miniprep kit and sequenced by Eurofins using a standard $\mathrm{T} 7$ terminal reverse primer. Afterward, the two different plasmids were transfected into Rosetta Gami 2 (DE3) pLysS competent cells (Novagen) and grown overnight on LB agar plates containing $34 \mathrm{mg} / \mathrm{L}$ chloramphenicol and $100 \mathrm{mg} / \mathrm{L}$ ampicillin. Individual colonies were grown in LB medium containing the mentioned antibiotics. For long-term storage, $15 \%$ glycerol bacterial stocks were made and stored at $-80{ }^{\circ} \mathrm{C}$.

Expression and Purification of (m)EETI-II Knottins. The expression and purification of EETI-II and mEETI-II knottins was performed according to the protocol by Sankaran et $\mathrm{al}^{13}$ As an alternative to the dialysis purification, size exclusion liquid chromatography (SEC) was performed using an ÄKTA purifier with a Frac-950 fractionation collector (GE Healthcare Life Sciences) (Figure S2C) equipped with a Superdex 75 10/300GL (GE Healthcare Life Sciences) column. Both methods gave comparable results. Concentrations of the knottins were determined from the absorbance $\varepsilon_{280 \mathrm{~nm}}=5875 \mathrm{M}^{-1} \mathrm{~cm}^{-1}$.

Binding of (m)EETI-II Knottins to $T$. maritima Encapsulin. The knottins were mixed with a freshly prepared sulfo-EMCS ( $N$ - $\varepsilon$-malemidocaproyl-sulfosuccinimide ester, Thermo Fisher) solution at a $50 \times$ molar excess for $1 \mathrm{~h}$ at room temperature. The unreacted sulfo-EMCS and side products were removed from the knottins using 3k MWCO centrifugal filters (Amicon Ultra) with $3 \times$ buffer refreshment (0.1× PBS, $0.1 \%(\mathrm{v} / \mathrm{v})$ Tween-20). TM encapsulin was rebuffered to the same buffer using Zeba Spin $7 \mathrm{k}$ MWCO Desalting Columns (Thermo Fisher). The concentrations of both knottins and encapsulins were measured with a nanodrop system, and the EMCS-knottins were reacted to the encapsulin at a $100 \times$ molar excess for at least $1 \mathrm{~h}$ at $21^{\circ} \mathrm{C}$. The unbound knottins were removed by applying a Zeba Spin 40k MWCO Desalting Column to the TM-mEETI-II solution twice.

SDS-PAGE Analysis of Trypsin Digestion of TM Encapsulin. A volume of $15 \mu \mathrm{L}$ of TM encapsulins, either with or without mEETI-II knottins attached to it, was added to $15 \mu \mathrm{L}$ of a $50 / 50$ mixture of $\beta$-mercaptoethanol and Laemmli sample buffer (Bio-Rad). The mixtures were incubated at 99 ${ }^{\circ} \mathrm{C}$ for $5 \mathrm{~min}$ to denature the proteins. The samples were analyzed with SDS-PAGE on a $4-20 \%$ gel (Bio-Rad). The unstained ladder was $10 \mu \mathrm{L}$ of Precision Plus Protein Standard (Bio-Rad). Electrophoresis was conducted at $100 \mathrm{~V}$ for $1 \mathrm{~h}$. Gels where activated with UV for $5 \mathrm{~min}$ on a stain-free enabled UV transilluminator and imaged with a Gel Doc EZ system with Image Lab software (Bio-Rad).

Trypsin Assay. TM encapsulins were rebuffered to $0.1 \times$ PBS + 0.05\% (v/v) TWEEN 20 with 7k MWCO Zeba Spin Columns. The samples were added to a flat-bottom 96-well 
plate (Greiner) in triplicate to a final concentration ranging from $0-1 \mu \mathrm{M}$. Trypsin was added to a final concentration of $0.1 \mathrm{mg} / \mathrm{mL}$. Immediately prior to analysis, $10 \mu \mathrm{L}$ of $\mathrm{N}_{\alpha}$ benzoyl-L-arginine 4-nitroanilide hydrochloride (L-BApNA) was added (final concentration $=142.2 \mu \mathrm{g} / \mathrm{mL}$ ). We measured the absorbance at $\lambda=405 \mathrm{~nm}$.

\section{RESULTS}

The production of TM encapsulins resulted in $\sim 20 \mathrm{~nm}$ sized nanoparticles as measured with DLS and FPLC (Figures S2A and $\mathrm{B}$ ). To verify that the thiol groups were available for binding, we reacted TM encapsulins with Oregon Green 488 maleimide-coupled dye. This was purified and subsequently analyzed using UV-vis. Using the peak at $\lambda=488 \mathrm{~nm}$, which corresponds to the Oregon Green 488 absorption, and $\varepsilon=$ $70000 \mathrm{M}^{-1} \cdot \mathrm{cm}^{-1}$, we determined the Oregon Green 488 concentration to be $2.49 \mu \mathrm{M}$. The peak at $\lambda=280 \mathrm{~nm}$ corresponding to TM encapsulins, which when corrected for the Oregon Green 488 using a correction factor of 0.12 at $\lambda=$ $280 \mathrm{~nm}$ and $\varepsilon=36565 \mathrm{M}^{-1} \cdot \mathrm{cm}^{-1}$, was used to calculate a TM encapsulin monomer concentration of $9.50 \mu \mathrm{M}$. This gives a degree of loading of $26.2 \%$, yielding $\sim 15$ dye molecules per encapsulin nanoparticle (Figure 1). This verified that the thiol

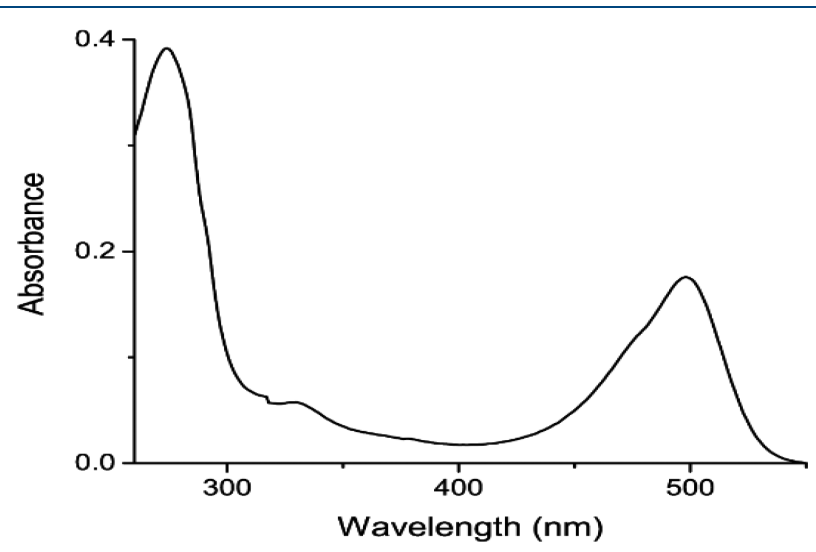

Figure 1. UV-vis spectrum of TM encapsulin with maleimidecoupled Oregon Green 488 dye attached to the exterior thiol groups.

groups on the exterior of TM encapsulins can be modified using maleimides. However, the coupling efficiency of Oregon Green 488 could not be increased beyond $26 \%$, most probably because the thiols from the cysteines that are accessible on the exterior, Cys 123, reside in a pocket ${ }^{21}$ (S3) resulting in less accessible thiol groups. Additionally, under the applied conditions, the cysteine sulfur might be partly oxidized, further limiting its reactivity.

To selectively bind the EETI-II knottin to TM encapsulin, we modified the EETI-II genetic sequence to remove a lysine from one of the loops to make the $\mathrm{N}$-terminus the only available binding site for bio-orthogonal binding with NHSester substitution. Furthermore, both the original and modified knottins where expressed in E. coli with an N-terminal flexible linker. To verify that the knottins were successfully formed and produced, we performed MALDI-TOF. For EETI-II knottin, the expected weight is $M=3589.1 \mathrm{Da}$, and the observed weight was $M=3590 \mathrm{Da}$. For mEETI-II, which was derived from EETI-II, but it has its lysine replaced with a serine (S1A, B), the expected weight is $M=3548 \mathrm{Da}$, and the observed weight was $M=3548.9 \mathrm{Da}$ (Figure S4). Thus, the EETI-II knottins were correctly produced and altered to mEETI-II. To determine the inhibitory capabilities of the knottins, they were mixed with trypsin and added to the L-BApNA substrate. Upon cleavage by trypsin, L-BApNA forms para-nitroaniline, and the absorption at $\lambda=405 \mathrm{~nm}$ increases. The mEETI-II knottin shows similar trypsin inhibition compared to the EETIII knottins (Figure 2A,B). The control sample (RGDknottin) ${ }^{26}$ did not inhibit trypsin (Figure 2C), while the trypsin inhibitor from bovine pancreas showed minor trypsin inhibition (Figure 2D).

We bound EMCS to the amine group of the mEETI-II knottins and reacted this with the TM encapsulins. Since the knottins cannot be distinguished with UV spectrometry when attached to the protein nanocage-both absorb at $\lambda=280 \mathrm{~nm}$, but the change in the extinction coefficient $(\varepsilon)$ of the knottins is not significant compared with the $\varepsilon$ of 60 encapsulin monomers - the main method of detection is by monitoring the trypsin inhibition capacity of the knottins (comparable to the experiments described in Figure 2). We found that TM encapsulins, without functionalization with knottins, already inhibit trypsin activity. Compared to TM, TM-mEETI-II showed enhanced but not complete inhibition within the concentration range studied, but the inhibition was not as significant as that of unbound mEETI-II. This is probably caused by steric hindrance of the encapsulin complex; the binding of a complex to knottins is known to decrease the knottin activity. ${ }^{13}$ After $1 \mathrm{~h}$ and at $1 \mu \mathrm{M}$, mEETI-II fully inhibits trypsin, TM encapsulins inhibit $32.43 \%$, and TMmEETI-II inhibits $64.33 \%$. At $0.5 \mu \mathrm{M}$, the difference between functionalized and nonfunctionalized TM encapsulin is significant $(P<0.001$ determined with an unpaired $t$ test $)$ (Figure S5). At lower concentrations, the effects of TM encapsulins, either functionalized or not, are not significantly different from those of the positive control.

We determined the rate of conversion based on the absorbance at $\lambda=405 \mathrm{~nm}$ using the slope in the linear range $(t=0-20 \mathrm{~min})$. These data were fitted with a sigmoidal fit with a dose response function using OriginPro 9.1. The $\mathrm{EC}_{50}$ of each compound was determined using this fit; the EC $_{50}$ for mEETI-II was $0.13 \mu \mathrm{M}$, for TM-mEETI-II, it was $0.73 \mu \mathrm{M}$, and for $\mathrm{TM}$, it was $>1 \mu \mathrm{M}$, but the data could not be fitted within this data set.

Equipping TM encapsulins with mEETI-II does not change the structure of the protein cages (Figure 3B). Both native TM and the TM-mEETI-II were treated with trypsin, which resulted in a loss of the structural integrity of for TM. Upon this treatment, the TM-mEETI-II does aggregate but keeps the individual cage structure (Figure $3 \mathrm{~B}$ ).

We performed SDS-PAGE to verify that no inhibition was caused by unbound mEETI-II in the TM-mEETI-II sample. The filtration steps were sufficient to remove unbound mEETIII from TM encapsulins, given the absence of a band at 3.5 $\mathrm{kDa}$ in the SDS-PAGE experiments (Figure S6).

\section{DISCUSSION}

In this study, we used TM encapsulins as a model to functionalize protein nanocages with EETI-II knottins. TM encapsulins can be functionalized specifically by binding compounds to the exterior cysteines, which we show are available for chemical binding (Figure 1). Sonotaki et al. have PEGylated Rhodococcus erythropolis N771 encapsulins, showing the potential for exterior modification. ${ }^{27}$ Specific functionalization has not been studied. Moon et al. genetically engineered a 

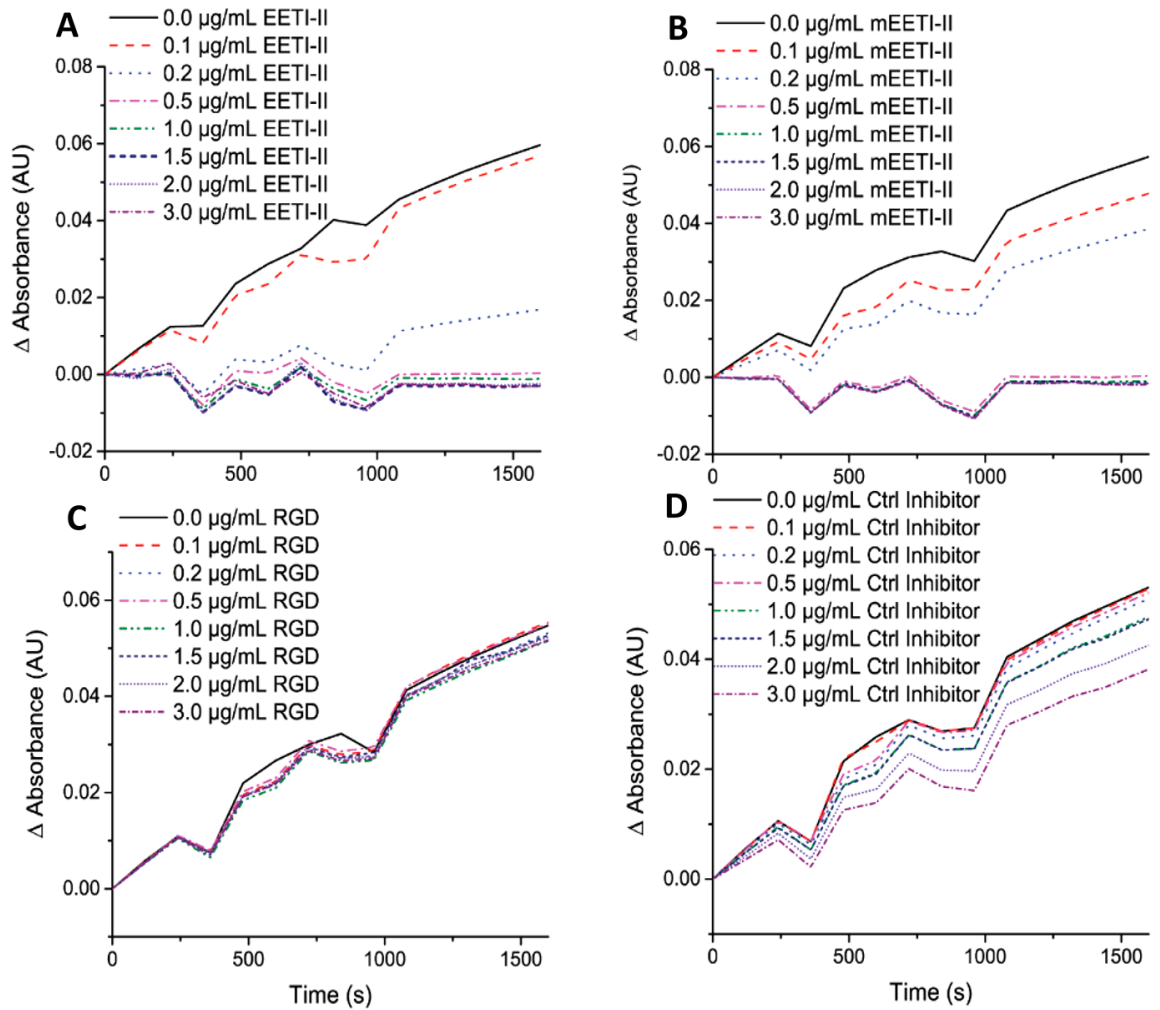

Figure 2. Difference in trypsin activity when inhibited by (A) EETI-II, (B) mEETI-II, (C) RGD-knottin, and (D) trypsin inhibitor from bovine pancreas. The $y$-axis indicates the difference in absorbance at $405 \mathrm{~nm}$ compared with $t=0$.
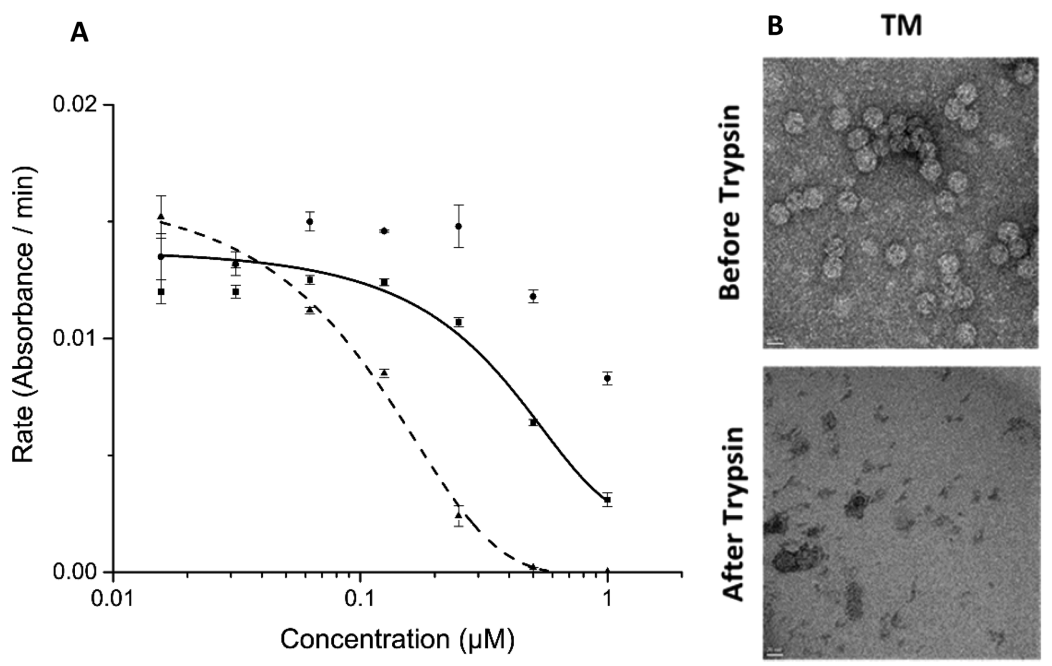

\section{TM-MEETI-II}

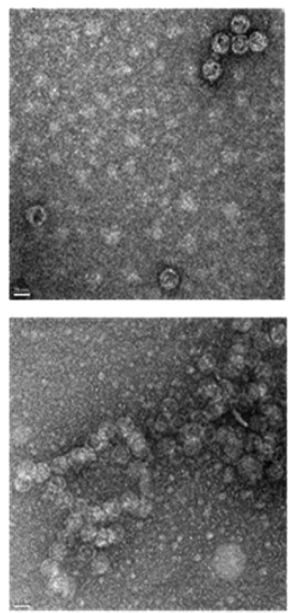

Figure 3. Inhibitory effects of TM-mEETI-II. (A) Rate of l-BApNA cleavage by trypsin in the presence of TM (circle), mEETI-II (triangle), and TM-mEETI-II (square). (B) TEM images of TM encapsulins with and without mEETI-II functionalization, before and after trypsin treatment. The scale bars represent $20 \mathrm{~nm}$.

targeting loop in TM encapsulins, ${ }^{24,28}$ but this method might be limited, because they altered the monomers forming the encapsulin shell.

We chemically modified the protein nanocage with the trypsin inhibiting knottin EETI-II. Prior to binding, the knottin requires modification to prevent nonspecific binding. The native EETI-II knottin contains a lysine ${ }^{15}$ that could bind the EMCS linker and connect the knottin to the encapsulin in such a way that the active loop cannot be accessed. Therefore, we modified the EETI-II knottin to replace the lysine with a nonreactive serine and extend the N-terminus with an SGSGS sequence to increase the length between the knottin and the encapsulin surface. As shown using the trypsin inhibition assay, these modifications were successful and did not affect the knottin activity (Figures 2A,B and S4).

Since EETI-II consists of 35 amino acids but contains no phenylalanine, tyrosine, or tryptophan, UV-vis detection is not possible, so we added a tryptophan to the C-terminus to both EETI-II and mEETI-II. This allowed for UV-vis detection when the knottin was cleaved from the TFP. However, when bound to TM encapsulin, which consists of 60 monomers of 268 amino acids, the single tryptophan is not 
sufficient to detect the presence of knottins attached to encapsulins. This makes it impossible to use UV-vis absorption to determine the number of knottins attached to one TM encapsulin.

Thus, the biological activity of EETI-II was used to determine if it was bound to TM encapsulins. Nonfunctionalized encapsulins have an inhibitory effect on trypsin (Figures 3A and S4), probably caused by competition between TM encapsulins and L-BApNA as substrate for trypsin. This $\mathrm{EC}_{50}$ is significantly smaller than TM encapsulins functionalized with mEETI-II. Here, the TM encapsulins show a significantly increased inhibition, which we attribute to the presence of mEETI-II, but they do not achieve a similar EC $_{50}$ as mEETI-II alone. Potentially, the bulk of the TM encapsulin limits the inhibitory effect of mEETI-II. This issue can potentially be overcome by using a longer linker, which results in a more available knottin.

The hypothesis that mEETI-II functionalized TM encapsulins are more stable is strengthened further by TEM analyses. These show that TM encapsulins are degraded when trypsin is added, while TM-mEETI-II keeps its individual morphological structure, but aggregates are formed (Figure 3B). This could be a result of interactions between the TM-encapsulinmEETI-II-trypsin complexes or hydrophobic interactions between TM encapsulins. The structural origin of this aggregation is at present under further investigation.

In conclusion, we show the possibility to functionalize a protein, in this case, the TM encapsulin nanoparticle, with mEETI-II knottins. We can modify these knottins without affecting their activity and use them to protect TM encapsulin from trypsin induced degradation. This concept can be applied to other nanocages and proteins to protect them from trypsin degradation. In addition, other knottins or other peptides with different functions can be added to create a heterofunctionalized protein nanocage, to allow, for example, specific targeting $^{29}$ and tumor suppression. ${ }^{30}$

\section{ASSOCIATED CONTENT}

\section{S Supporting Information}

The Supporting Information is available free of charge on the ACS Publications website at DOI: 10.1021/acs.molpharmaceut.8b00630.

The plasmid and gene information on TM and ( $\mathrm{m})$ EETI-II is presented as well as FPLC, DLS, and MALDI-TOF data on the structure of TM and (m)EETI-II. Additionally, the SI includes the SDSPAGE of TM encapsulin with and without mEETI-II attached $(\mathrm{PDF})$

\section{AUTHOR INFORMATION}

\section{Corresponding Author}

*E-mail: j.j.l.m.cornelissen@utwente.nl.

\section{ORCID}

Jeroen J.L.M. Cornelissen: 0000-0002-9728-5043

\section{Author Contributions}

${ }^{\dagger}$ R.K. and M.V.d.R. contributed equally to this paper.

\section{Notes}

The authors declare no competing financial interest.

\section{ACKNOWLEDGMENTS}

We thank R. van der Hee for her help with the MALDI-TOF experiment. We also thank Dr. W.F. Rurup for providing the plasmids and bacterial cryo stock to produce TM encapsulin. The European Research Council (ERC) is acknowledged for financial support via the Consolidator Grant "Protcage" (616907).

\section{REFERENCES}

(1) Nascimento, I. P.; Leite, L. C. C. Recombinant Vaccines and the Development of New Vaccine Strategies. Braz. J. Med. Biol. Res. 2012, 45, 1102-1111.

(2) Pieters, R.; Hunger, S. P.; Boos, J.; Rizzari, C.; Silverman, L.; Baruchel, A.; Goekbuget, N.; Schrappe, M.; Pui, C. L-asparaginase Treatment in Acute Lymphoblastic Leukemia: a Focus on Erwinia Asparaginase. Cancer 2011, 117, 238-249.

(3) Kirkegaard, T.; Gray, J.; Priestman, D. A.; Wallom, K. L.; Atkins, J.; Olsen, O. D.; Klein, A.; Drndarski, S.; Petersen, N. H.; Ingemann, L.; Smith, D. A.; Morris, L.; Bornæs, C.; Jørgensen, S. H.; Williams, I.; Hinsby, A.; Arenz, C.; Begley, D.; Jäättelä, M.; Platt, F. M. Heat Shock Protein-based Therapy as a Potential Candidate for Treating the Sphingolipidoses. Sci. Transl. Med. 2016, 8, 355ra118.

(4) Solá, R. J.; Griebenow, K. Glycosylation of Therapeutic Proteins: An Effective Study to optimize Efficacy. BioDrugs 2010, 24, 9-21.

(5) Getz, J. A.; Rice, J. J.; Daugherty, P. S. Protease-resistant Peptide Ligands from a Knottin Scaffold Library. ACS Chem. Biol. 2011, 6, 837-844.

(6) Kolmar, H. Alternative Binding Proteins: Biological Activity and Therapeutic Potential of Cystine-knot Miniproteins. FEBS J. 2008, $275,2684-2690$

(7) Gelly, J.-C.; Gracy, J.; Kaas, Q.; Le-Nguyen, D.; Heitz, A.; Chiche, L. The KNOTTIN Website and Database: a New Information System Dedicated to the Knottin Scaffold. Nucleic Acids Res. 2004, 32, D156-D159.

(8) Gracy, J.; Le-Nguyen, D.; Gelly, J. C.; Kaas, Q.; Heitz, A.; Chiche, L. KNOTTIN: the Knottin or Inhibitor Cystine Knot Scaffold in 2007. Nucleic Acids Res. 2008, 36, D314-D319.

(9) Souza, A. H.; Ferreira, J.; Nascimento Cordeiro, M. d.; Vieira, L. B.; De Castro, C. J.; Trevisan, G.; Reis, H.; Souza, I. A.; Richardson, M.; Prado, M. A.; et al. Analgesic Effect in Rodents of Native and Recombinant $\mathrm{Ph}$ Alpha 1beta Toxin, a High-voltage-activated Calcium Channel Blocker Isolated from Armed Spider Venom. Pain 2008, 140, 115-126.

(10) Park, S. P.; Kim, B. M.; Koo, J. Y.; Cho, H.; Lee, C. H.; Kim, M.; Na, H. S.; Oh, U. A Tarantula Spider Toxin, GsMTx4, Reduces Mechanical and Neuropathic Pain. Pain 2008, 137, 208-217.

(11) Choi, S. J.; Parent, R.; Guillaume, C.; Deregnaucourt, C.; Delarbre, C.; Ojcius, D. M.; Montagne, J. J.; Célérier, M. L.; Phelipot, A.; Amiche, M.; Molgo, J.; Camadro, J. M.; Guette, C. Isolation and Characterization of Psalmopeotoxin I and II: Two Novel Antimalarial Peptides from the Venom of the Tarantula Psalmopoeus Cambridgei. FEBS Lett. 2004, 572, 109-117.

(12) Gould, A.; Ji, Y.; Aboye, T. L.; Camarero, J. A. Cyclotides, a Novel Ultrastable Polypeptide Scaffold for Drug Discovery. Curr. Pharm. Des. 2011, 17, 4294-4307.

(13) Sankaran, S.; de Ruiter, M.; Cornelissen, J. J.; Jonkheijm, P. Supramolecular Surface Immobilization of Knottin Derivatives for Dynamic Display of High Affinity Binders. Bioconjugate Chem. 2015, 26, 1972-1980.

(14) Moore, S. J.; Leung, C. L.; Cochran, J. R. Knottins: Disulfidebonded Therapeutic and Diagnostic Peptides. Drug Discovery Today: Technol. 2012, 9, e3-e11.

(15) Kintzing, J. R.; Cochran, J. R. Engineered Knottin Peptides as Diagnostics, Therapeutics, and Drug Delivery Vehicles. Curr. Opin. Chem. Biol. 2016, 34, 143-150.

(16) Bode, W.; Greyling, H. J.; Huber, R.; Otlewski, J.; Wilusz, T. The Refined 2.0 A X-ray Crystal Structure of the Complex Formed Between Bovine beta-trypsin and CMTI-I, a Trypsin Inhibitor from 
Squash Seeds (Cucurbita maxima). Topological Similarity of the Squash Seed Inhibitors with the Carboxypeptidase A Inhibitor from Potatoes. FEBS Lett. 1989, 242, 285-292.

(17) Krätzner, R.; Debreczeni, J. E.; Pape, T.; Schneider, T. R.; Wentzel, A.; Kolmar, H.; Sheldrick, G. M.; Uson, I. Structure of Ecballium Elaterium Trypsin inhibitor II (EETI-II): a Rigid Molecular Scaffold. Acta Crystallogr., Sect. D: Biol. Crystallogr. 2005, 61, 12551262.

(18) Schmoldt, H.-U.; Wentzel, A.; Becker, S.; Kolmar, H. A Fusion Protein System for the Recombinant Production of Short Disulfide Bond Rich Cystine Knot Peptides Using Barnase as a Purification Handle. Protein Expression Purif. 2005, 39, 82-89.

(19) Azizgolshani, O.; Garmann, R. F.; Cadena-Nava, R.; Knobler, C. M.; Gelbart, W. M. Reconstituted Plant Viral Capsids can Release Genes to Mammalian Cells. Virology 2013, 441, 12-17.

(20) O’Neil, A.; Reichhardt, C.; Johnson, B.; Prevelige, P. E.; Douglas, T. Genetically Programmed in Vivo Packaging of Protein Cargo and its Controlled Release from Bacteriophage P22. Angew. Chem., Int. Ed. 2011, 50, 7425-7428.

(21) Yang, Y.; Burkhard, P. Encapsulation of Gold Nanoparticles into Self-assembling Protein Nanoparticles. J. Nanobiotechnol. 2012, 10,42 .

(22) Sutter, M.; Boehringer, D.; Gutmann, S.; Günther, S.; Prangishvili, D.; Loessner, M. J.; Stetter, K. O.; Weber-Ban, E.; Ban, N. Structural Basis of Enzyme Encapsulation into a Bacterial Nanocompartment. Nat. Struct. Mol. Biol. 2008, 15, 939-947.

(23) Cassidy-Amstutz, C.; Oltrogge, L.; Going, C. C.; Lee, A.; Teng, P.; Quintanilla, D.; East-Seletsky, A.; Williams, E. R.; Savage, D. F. Identification of a Minimal Peptide Tag for in Vivo and in Vitro Loading of Encapsulin. Biochemistry 2016, 55, 3461-3468.

(24) Moon, H.; Lee, J.; Min, J.; Kang, S. Developing Genetically Engineered Encapsulin Protein Cage Nanoparticles as a Targeted Delivery Nanoplatform. Biomacromolecules 2014, 15, 3794-3801.

(25) Rurup, W. F.; Snijder, J.; Koay, M. S.; Heck, A. J.; Cornelissen, J. J. Self-sorting of Foreign Proteins in a Bacterial Nanocompartment. J. Am. Chem. Soc. 2014, 136, 3828-3832.

(26) Sankaran, S.; Cavatorta, E.; Huskens, J.; Jonkheijm, P. Cell Adhesion on RGD-Displaying Knottins with Varying Numbers of Tryptophan Amino Acids to Tune the Affinity for Assembly on Cucurbit[8] uril Surfaces. Langmuir 2017, 33, 8813-8820.

(27) Sonotaki, S.; Takami, T.; Noguchi, K.; Odaka, M.; Yohda, M.; Murakami, Y. Successful PEGylation of Hollow Encapsulin Nanoparticles from Rhodococcus Erythropolis N771 without Affecting Their Disassembly and Reassembly Properties. Biomater. Sci. 2017, 5, 1082-1089.

(28) Moon, H.; Lee, J.; Kim, H.; Heo, S.; Min, J.; Kang, S. Genetically Engineering Encapsulin Protein Cage Nanoparticle as a SCC-7 Cell Targeting Optical Nanoprobe. Biomater. Sci. 2014, 18, 21.

(29) Kimura, R. H.; Cheng, Z.; Gambhir, S. S.; Cochran, J. R. Engineered Knottin Peptides: A New Class of Agents for Imaging Integrin Expression in Living Subjects. Cancer Res. 2009, 69, 24352442.

(30) Treggiari, D.; Zoccatelli, G.; Molesini, B.; Degan, M.; Rotino, G. L.; Sala, T.; Cavallini, C.; MacRae, C. A.; Minuz, P.; Pandolfini, T. A Cystine-knot Miniprotein from Tomato Fruit Inhibits Endothelial Cell Migration and Angiogenensis by Affecting Vascular Endothelial Growth Factor Receptor (VEGFR) Activation and Nitric Oxide Production. Mol. Nutr. Food Res. 2015, 59, 2255-2266. 